\title{
ESTIMATIVA DA VARIABILIDADE DE PRODUÇÃO DE ENERGIA UTILIZANDO DADOS MEDIDOS E MODELADOS
}

\author{
Ana Carolina Cardoso Pereira' ${ }^{1}$ Marcos Miranda ${ }^{2}$ \\ ${ }^{12}$ Inova Energy
}

\section{RESUMO}

O objetivo deste trabalho foi verificar a variabilidade da produção de energia calculada a partir de dados simulados ao longo de 10 anos, com o modelo WRF (Weather Research and Forcasting). Os resultados da torre anemométrica do Rio Grande do Sul (TA RS) mostram um índice de correlação $\mathrm{r}=0,90$ entre a série medida e a simulada, a $80 \mathrm{~m}$ de altura no período de maio de 2009 a março de 2013. Para a torre anemométrica do Rio Grande do Norte (TA RN) o índice de correlação foi $\mathrm{r}=0,78$. A variabilidade da produção de energia em torno da média é menor na TA RS.

\section{ABSTRACT}

The objective of this study was to determine the variability of energy production calculated from simulated data over 10 years, with the WRF (Weather Research and Forcasting) model. The results of the anemometer tower of Rio Grande (TA RS) a correlation coefficient index $\mathrm{r}=0,90$ between measured and simulated series, the $80 \mathrm{~m}$ in the period May 2009 to March 2013. For the anemometer tower of Pedra Grande (TA RN) correlation index was $r=0,78$. The variability of energy production around the average TA is lower in RS.

\section{INTRODUÇÃO}

O estudo da variabilidade do vento é fundamental para aviação, agricultura e energia (Oliveira et al, 2012). A energia eólica está diretamente relacionada ao cálculo de produção de energia, sendo assim torna-se indispensável conhecer os padrões de vento ao longo prazo. Atualmente, apesar da grande expansão da energia eólica no Brasil, os dados de velocidade e direção do vento para períodos maiores que cinco anos são escassos. Desta forma, inúmeras técnicas vêm sendo aprimoradas para melhor estimar a velocidade do vento em períodos não medidos, entre elas: probabilidade condicional, redes neurais, MCP "measue-correlatedpredict”. Outra metodologia bastante difundida é a utilização de dados simulados através de modelos atmosféricos.

Neste contexto, este estudo tem o objetivo de verificar a variabilidade da produção de energia obtida a partir de dados simulados e assim observar a representatividade dos ventos simulados ao longo do tempo.

\section{MATERIAL E MÉTODOS}

Para este trabalho utilizaram-se dados de velocidade e direção do vento, a $80 \mathrm{~m}$ de altura, de duas torres anemométricas localizadas no Rio Grande do Sul/RS e Rio Grande do Norte/RN. Estes registros são médias de 10 em 10 minutos, obtidos por um anemômetro de copo e uma windvane no período de maio de 2009 a março de 2013. A partir destes dados, foram 
calculadas médias horárias e mensais para caracterizar os ventos locais e estabelecer comparações com séries simuladas com o modelo WRF nos respectivos locais.

A simulação dos dados de velocidade do vento, utilizando modelo de mesoescala WRF, consiste em uma série temporal de 10 anos de duração com dados horários e resolução espacial de $3 \mathrm{~km}$, fornecida pela Vortex ${ }^{1}$. Esse conjunto de dados foi correlacionado com os dados observados e ajustado a partir da razão entre a média anual simulada e a média anual observada para cada um dos locais separadamente.

Finalizado o ajuste da série simulada de 10 anos, foi feita uma estimativa de produção de energia com uma turbina hipotética de 1,6MW no software Windographer 3.0.11.

\section{RESULTADOS}

Para TA RS, obteve-se uma boa representatividade entre a série medida e a série simulada, no período de maio de 2009 a março de 2013, o índice de correlação foi r=0,90. As médias, medida e simulada para o período, foram de $6,87 \mathrm{~m} / \mathrm{s}$ e $6,83 \mathrm{~m} / \mathrm{s}$, respectivamente. Já para a TA RN, o índice de correlação foi $\mathrm{r}=0,78$ e as médias anuais medida e simulada foram de $7,98 \mathrm{~m} / \mathrm{s}$ e $8,23 \mathrm{~m} / \mathrm{s}$. A razão entre as médias simulada e medida de ambas as torres foi de 1,010 (TA RS) e 1,037 (TA RN). Esses valores são multiplicadores das séries simuladas para realizar o ajuste dos dados simulados e assim melhor representar os ventos de cada região.

A Figura 1 apresenta a velocidade média mensal do vento medido e simulado ajustado na TA RS, no período de maio de 2009 a março de 2013. Os valores de velocidade do vento superestimados e subestimados não apresentaram um padrão comum, ocorrendo em qualquer mês do ano.

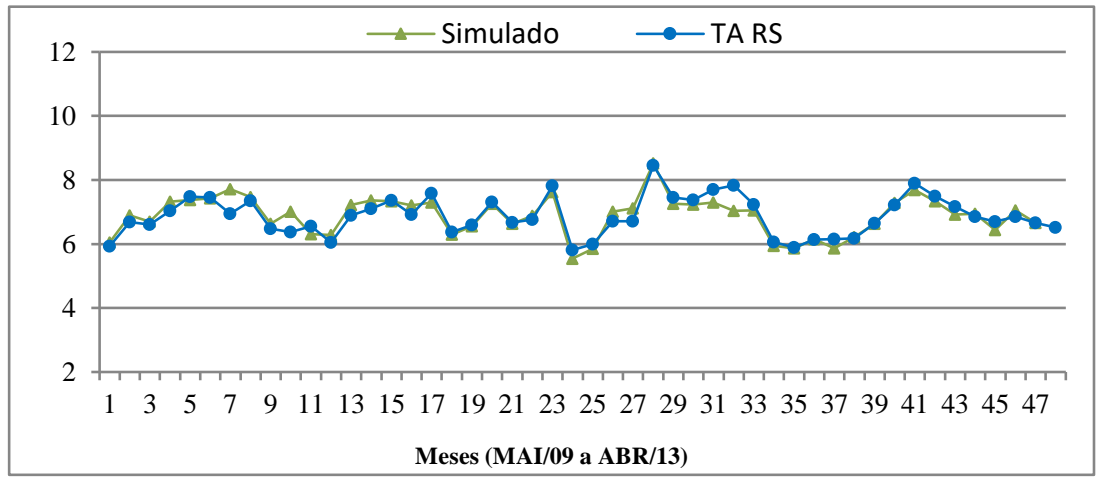

Figura 1: Velocidade média mensal do vento medida e simulada, no período de maio de 2009 a março de 2013 - TA RS.

O ajuste da série simulada e as médias mensais medidas da TA RN são apresentados na Figura 2. Contrária a TA RS, nota-se um padrão quando os dados são superestimados, fato que ocorre nos meses de maio a setembro. 


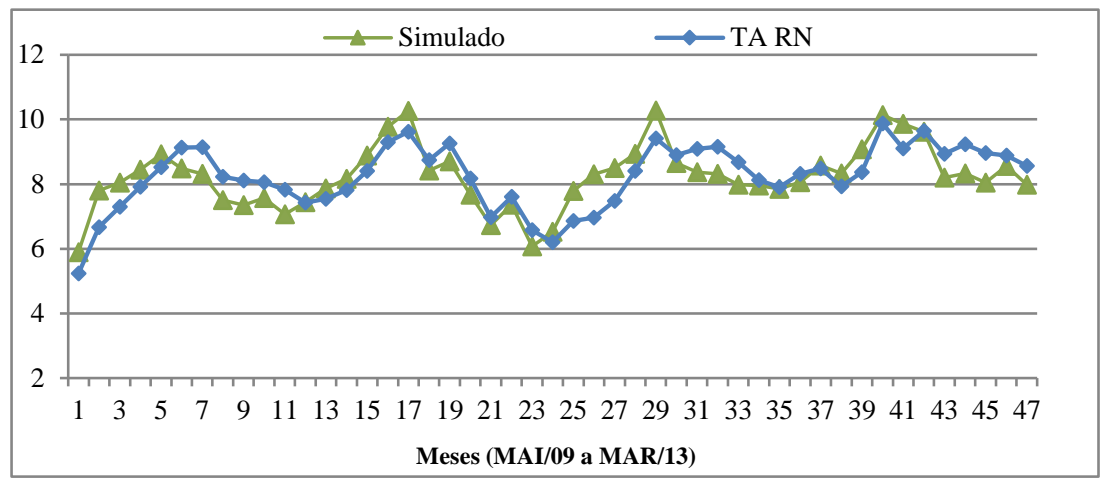

Figura 2: Velocidade média mensal do vento medida e simulada, no período de maio de 2009 a abril de 2013 - TA RN.

A Figura 3 mostra os resultados obtidos após o calculo da produção de energia ao longo de 10 anos para as duas localidades. Observa-se, pelo formato senoidal, uma sazonalidade melhor definida na TA RN, ao contrario da TA RS onde tais variações são se forma aleatória. Embora os máximos de potência estejam semelhantes, em torno de $40 \%$ acima da média, os mínimos são mais expressivos, quase $65 \%$ abaixo da média no nordeste. Quanto ao desvio padrão, este é menor no sul (16\%) que no nordeste (23\%).

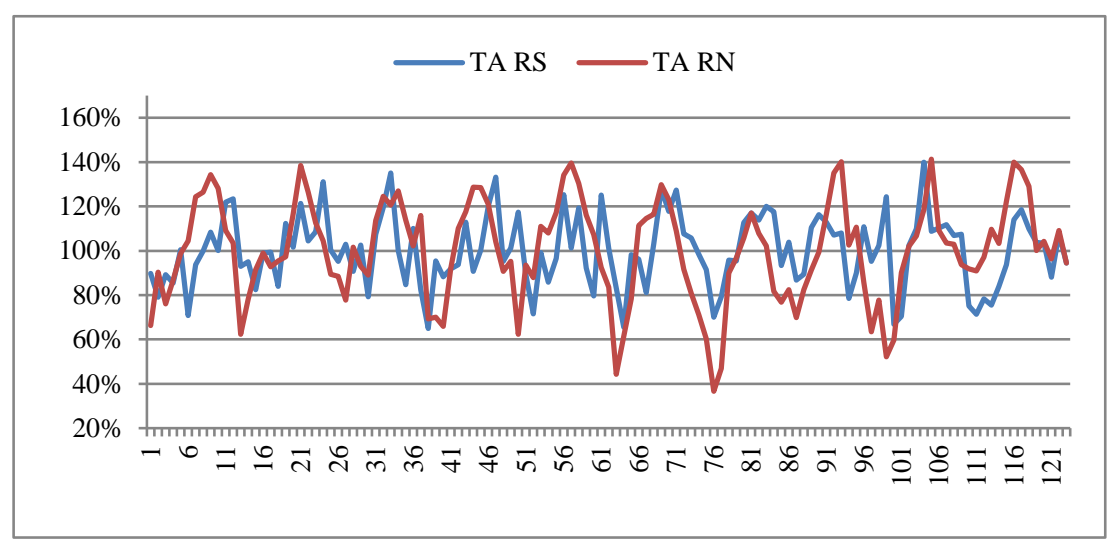

Figura 3: Produção normalizada de energia ao longo de 10 anos no Rio Grande do Sul e Rio Grande do Norte.

\section{CONCLUSÕES}

Por fim, os bons índices de correlação entre os dados medidos e simulados não são suficientes para comprovar a adequabilidade dos dados de vento simulados no calculo de produção de energia. Sendo assim, um maior número de torres anemométricas deverá ser considerado em trabalhos futuros.

\section{BIBILIOGRAFIA}

OLIVEIRA, C.S.; FERREIRA, C.C.M.. Caracterização da direção e velocidade do vento para os meses de agosto de 2010 a julho de 2011 em Juiz de Fora - MG. In: XVII Congresso Brasileiro de Meteorologia, 2012, Gramado. 